\title{
DEL INSOMNIO EN BLANCHOT AL QUIASMO EN MERLEAU-PONTY: FIGURAS DEL EXCESO
}

\author{
From Insomnia in Blanchot to Chiasm in Merleau-Ponty: figures of excess \\ Paulina Morales Guzmán \\ Universidad Diego Portales, Santiago, Chile \\ plmorales@uc.cl
}

\section{Resumen}

El presente trabajo consiste en una confrontación entre las propuestas de Maurice Merleau-Ponty y Maurice Blanchot, con el objetivo de determinar el lugar que ocupa en el pensamiento de ambos autores la crítica al subjetivismo en la expresión artística. Lo anterior se hace manifiesto en el planteamiento de un modo de existencia que desafía la constitución de un sujeto, mediante la introducción de un exceso que escapa a la interioridad subjetiva. Para ello se desarrollará, en primer lugar, la propuesta blanchoteana del insomnio para luego vincularla con su planteamiento en torno a la escritura a la luz de una suspensión del sujeto. Luego, se abordará la propuesta de MerleauPonty sobre el quiasmo en la sensibilidad del cuerpo, reconociendo movimientos argumentales afines entre ambos pensamientos. Realizado lo anterior, se pretende plantear una nueva forma de comprender la expresión artística en función de las propuestas que ambos autores tienen del exceso.

Palabras clave: insomnio, quiasmo, cuerpo, expresión, literatura.

\begin{abstract}
The present essay consists of a confrontation between the proposals of Maurice Merleau-Ponty and Maurice Blanchot, with the aim of determining the place that the criticism of subjectivism in artistic expression occupies in the thinking of both authors. The foregoing is manifested in the approach of a mode of existence that challenges the constitution of a subject from the presence of an excess that escapes the subjective interiority. To this end, firstly, it will be analyzed the Blanchotean proposal of 'insomnia' and then linked with its approach to writing towards a suspension of the subject. Then, Merleau-Ponty's proposal on chiasm in body sensitivity will be addressed, recognizing related plot movements between both thoughts. Having done the above, a new way of understanding artistic expression will be recognized based on the proposals that both authors have of the excess.
\end{abstract}

Keywords: insomnia, chiasm, body, expression, literature.

Fecha de Recepción: 06/08/2019 - Fecha de Aceptación: 20/12/2019 


\section{Introducción}

El pensamiento de Maurice Blanchot y Maurice Merleau-Ponty se encuentran en el importante rol que ocupa el análisis de expresiones artísticas en ellos. Pero ambos, en principio, construyen propuestas filosóficas diferentes: Blanchot piensa el mundo y la literatura desde una forma propia de entender la consciencia, en cambio, Merleau-Ponty hereda una formación fenomenológica que luego reformula a partir del cuerpo sensible, lo que le permite explorar la expresividad del mismo. A pesar de dichas diferencias, ambos autores piensan el sentido del texto literario más allá de la correspondencia entre signo y significado ${ }^{1}$, considerando en sus propuestas los vacíos y la negatividad de lo que se muestra, rechazando la significación por mera correspondencia de signos. Blanchot piensa la literatura como aquello que no depende de una subjetividad, propuesta que le brinda a la escritura un estatuto diferente al de una mera producción artística. Asimismo, Merleau-Ponty en sus trabajos tardíos elabora una concepción del cuerpo que sugiere una suspensión del sujeto, y con ello, una propuesta de la expresión similar a aquella que Blanchot elabora con respecto a la literatura.

En virtud tanto de las intuiciones comunes entre ambos autores como de las diferencias metodológicas de cada uno se hace menester ahondar en las potenciales similitudes y afinidades de sus propuestas. Por ello, este trabajo busca establecer algo más que un punto común: se busca constatar un movimiento afín en sus propuestas, proponiendo que la crítica a la oposición entre sujeto-objeto y pasividad-actividad es lo que guía ambos pensamientos. En función de este objetivo, se busca rescatar la noción de sujeto que se presenta en la literatura según Blanchot, resaltando la figura del insomnio en El espacio literario, que se confrontará con el sujeto que Merleau-Ponty plantea en Lo visible y lo invisible a partir de la idea del quiasmo. De esta forma, esta investigación pretende reflexionar en torno a la subjetividad en el ejercicio de la creación artística, notando que dicha noción toma un giro a partir de los planteamientos de los autores ya señalados. Así, se destacará la problematización que ambos autores llevan a cabo en la relación entre sujeto y mundo, mostrando cómo ella permite pensar la expresión artística desde una nueva perspectiva.

\footnotetext{
${ }_{1}$ Dicha correspondencia plantea que el sentido de lo escrito queda determinado por el signo inscrito, habiendo entonces una relación directa entre el signo, como referente y causa, y una significación establecida por el anterior.
} 


\section{La pasividad y la actividad en el insomnio blanchoteano}

Blanchot, en su ensayo titulado "El dormir, la noche", presente en El espacio literario, reflexiona en torno a la experiencia del día y de la noche, más particularmente, a partir de la economía que subyace a ambas instancias, manifestándose en una codependencia entre ellos.

¿Qué pasa durante la noche? En general dormimos. Por el dormir, el día se sirve de la noche para borrar la noche. Dormir pertenece al mundo, es una tarea, dormimos de acuerdo con la ley general que hace depender nuestra actividad diurna del reposo de nuestras noches (Blanchot 2002 235).

Cuando visibiliza la codependencia que hay entre día y noche, Blanchot presenta una dualidad que, en suma, solo sirve a la lógica de uno: el día. En efecto, la noche pensada desde la experiencia del dormir es solo una reserva al servicio del día, en la medida en que dormir implica la suspensión de la noche en vistas de posibilitar una plena experiencia del día. Por ello, se afirma que la noche, a través del dormir, es pensada desde una lógica diurna. Ahora bien, lo interesante es que Blanchot realiza esta reflexión presentando el dormir desde la lógica de la consciencia y del control del actuar, en tanto que dormir es descrito como "una tarea" que se realiza de acuerdo con una "ley general", la de poder realizar una actividad diurna. En efecto, la lógica del día está bosquejada como el orden que determina el actuar de la consciencia en la noche a través del dormir. Por ello, Blanchot se cuestiona quién duerme: "Yo duermo, la soberanía del "Yo" domina esta ausencia que ella se concede y que es su obra" (2002 235). El dormir, como suspensión de la consciencia diurna, lo que finalmente suspende son sus interacciones con el mundo, encerrándose en un Yo tan inaccesible como aquel que domina en las actividades diurnas. Blanchot describe esta inaccesibilidad como "concentrada en la estrechez de ese lugar donde el mundo se recoge [...]" (Id. 236), concentración que debe leerse en su misma etimología: como una con-centración del mundo y del Yo en un eje delimitado por la suspensión del dormir -es decir, que la suspensión implica un Yo desconectado de la interpelación del mundo, dando lugar a una absoluta unidad con él. Esta normatividad del día determina un estado de reposo en el que la consciencia se halla completamente desligada de lo exterior y que le permite sostener a su opuesto: el absoluto ensimismamiento de una consciencia yoica frente al mundo.

En vista de esta lógica diurna del ir "del día a la noche" (Blanchot 2002 237), Blanchot presenta un ir de la noche al día. Para ello introduce una nueva experiencia de la noche como insomnio: "Si no dormimos, el final el agotamiento nos infecta; esta infección impide dormir, se traduce por el insomnio, [...]." (Ibid.). Blanchot alude a una infección, a un inmiscuir de algo indefinido en la experiencia 
de la noche. Esta invasión impide la con-centración con el mundo y se vuelve, más bien, una diseminación de aquello que debía ser un Yo suspendido. La figura del insomnio ilustra la instancia de la consciencia en la que ya no se encuentra en la absoluta pasividad, debido a que ya no hay con-centración como la completa unión con el mundo (Id. 236); pero tampoco implica una absoluta actividad, debido a que el insomnio desafía -sin pretensión de hacerlo- la normatividad que la lógica diurna dictamina sobre la experiencia de la noche. El insomnio ilustra, entonces, una consciencia que no es ni completamente ajena del mundo, ni completamente unida al mismo; con ello, se presenta una forma de consciencia dispersa, es decir, ya no basada en un Yo fundacional.

Un último elemento introducido es el sueño: “Quien sueña duerme, pero el que sueña ya no es más el que duerme, no es otro, otra persona, es el presentimiento de lo otro, lo que ya no puede decir yo, lo que no reconoce ni en sí ni en otros" (Blanchot 2002 238). Aquí se alude a una instancia en la cual el Yo no se suspende, sino que desaparece. Si el dormir suspendía al sujeto para dar lugar a su posterior despertar en el día, soñar implica un despertar que no es diurno y que no se vale de un previo reposo. El sueño es la experiencia del día, en la medida en que hay vivencia de una forma de presentarse del mundo, tal como la vigilia del día; no obstante, esta experiencia se configura fuera de la normatividad del $\mathrm{Yo}^{2}$, ya que las experiencias en el sueño subvierten la normatividad de la vigilia, presentando una nueva configuración del mundo en la que este ya no es dominado desde un Yo central, sino que está inmiscuido entre lo propio y lo ajeno. En suma, el sueño es una forma de insomnio, en tanto que es una experiencia nocturna ${ }^{3}$ del dormir -es decir, ya no experimentada en virtud de un día por venir. En el sueño la unión con el mundo no prima, sino una inquietud que se transfigura en una experiencia fuera del estado de vigilia. Esta consciencia inquieta implica estados de dispersión y de inmersión simultáneas con el mundo.

Ahora bien, el sueño y el insomnio designan instancias de la consciencia específicas, cuya particularidad puede apreciarse de manera tácita en la indagación blanchoteana sobre la literatura y, más precisamente, en el gesto de la escritura. En efecto, en "La literatura y el derecho a la muerte" Blanchot realiza, entre muchas reflexiones, un análisis de lo que es la literatura como cuestión y su relación con lo normativo. Ante todo, una de las nociones que el autor nos entrega de entrada al texto es la idea de una escritura que es una existencia en sí misma. Esto quiere decir que ella no responde a una lógica causal, ante lo cual, el que haya escritura, el

\footnotetext{
2 Entendiendo esta normatividad como lo dictaminado por el Yo, el 'deber ser' instaurado desde un Yo separado del mundo -como se le ha entendido desde las experiencias del insomnio y del sueño ya expuestas.

${ }^{3}$ En el sentido blanchoteano antes expuesto.
} 
hecho de que exista, no se debe a una subjetividad o a una objetividad operando como causas. La escritura no se reduce a sujeto o a objeto porque ella existe de un modo diferente a aquel que define las existencias causadas. La idea de una escritura como existencia en sí misma se puede obtener a partir de la manera en que el primer párrafo está planteado, en donde se propone un cuestionamiento por parte del escritor a su escritura: “ ¿Tiene un escritor, que mira cómo su pluma traza letras, el derecho de dejarla en suspenso y decirle: ¡Detente! Qué sabes de ti misma?" (Blanchot 2007 271). De suponer que la escritura no es una existencia en sí, sino una actividad directamente significante desde signos dispuestos por un polo subjetivo, a su vez se supondría un derecho del autor por sobre una escritura que es enteramente suya. En su lugar, Blanchot plantea esta pregunta justamente para evidenciar que es problemático pensar la escritura como un ejercicio que tenga toda su determinación en un sujeto unilateral: la falta de derecho de un autor por sobre su escritura implica pensar la literatura de un modo diferente.

Como ya se afirmó, el escritor, para Blanchot, no se entiende como punto genético desde el cual el texto literario cobra un sentido, sino que es también causado por la misma existencia de la escritura: "Mientras, no habiéndose sentado en la mesa, no haya escrito una obra, el escritor no es escritor y no sabe si tiene capacidades para llegar a serlo" (2007 273). El ser escritor, para Blanchot, es diferente de ser un sujeto yoico, ya que se es escritor en virtud de la relación del mismo con la escritura. Este escritor ya no tiene un poder sobre su escritura, sino que él es parte de la misma, lo que implica que se mueve con ella en lugar de posicionarse como un externo que la enfrenta. La escritura del texto literario, entonces, subvierte la necesidad de un origen para ser planteada como una instancia: “¿por qué no ves que tu tinta no deja huellas, que te adelantas libremente, pero en el vacío, que si no encuentras obstáculo es porque nunca has abandonado tu punto de partida?" (Id. 271). La escritura como instancia se ve reflejada en una escritura que "no deja huellas", esto quiere decir que aquel conjunto material de signos está abierto a una constante re-presentación (en tanto que nuevas instancias de presentación). Con ello, el texto literario solo puede afirmarse como vacío, indeterminado, pero que sí presenta una emergencia en la medida en que es un "punto de partida" permanente que carece de un fin al cual tiende.

Ahora bien, ¿cómo debe pensarse al sujeto que es escritor? El texto literario, tal como Blanchot nos lo presenta, ya no puede considerarse como parte de la lógica diurna, ya que este no supone una pre-determinación de su sentido desde un sujeto. Luego, el sujeto de la escritura (que deviene escritor) debe pensarse dentro de la lógica nocturna, de aquella noche que no está al servicio del día, sino que se piensa en la presencia misma de la noche: es decir, un sujeto que no se encuentra concentrado, sino disperso y paradójicamente interpelado por un 
mundo que se inmiscuye en su existencia; un escritor desde el insomnio. Entonces, se está pensando en una forma de sujeto que, paradójicamente, ya se despoja de su calidad de sujeto, en la medida en que su límite que lo separa del mundo es difuso y a la vez presente. En el insomnio existe una perplejidad que hace al sujeto aún más presente en su situación en el mundo, pero, a su vez, desposeído de un centro yoico.

\section{El quiasmo en Merleau-Ponty y la suspensión del sujeto}

Esta forma no subjetiva de situarse en el mundo, tal como plantea Blanchot, puede tener su punto de encuentro con la concepción que Merleau-Ponty desarrolla sobre el cuerpo a lo largo de sus obras. Dicho autor busca establecer la posibilidad de exceder la categoría de sujeto (y con ella, la de objeto), planteando, en cambio, una ontología que se base en la unión de ambas esferas tradicionalmente demarcadas, unión desembocada en el cuerpo. El cuerpo, para Merleau-Ponty, ya no se comprende desde su acepción cósica para un ego cogito, sino que es pensado en virtud de su potencialidad sensible, sensibilidad que permite desdibujar la frontera que separa al sujeto del mundo, estableciendo en su lugar una continuidad. Ya desde su texto temprano Fenomenología de la Percepción es posible constatar en su trabajo una pretensión de entender el cuerpo desde su carnalidad que vincula las esferas sujeto y objeto, tal como Barbaras lo observa en The Being of the Phenomenon: "[El cuerpo] aparece como el aún misterioso lugar donde la relación sujeto-objeto está unida" (Barbaras 7; traducción e inserción propia). En efecto, Merleau-Ponty lleva a cabo su propuesta del cuerpo en el marco de una crítica a la hipótesis del intelectualismo que pretende reducir la experiencia vivida a constituciones de una consciencia (Id. 5).

Ahora bien, el problema de esta consciencia es que supone ser un espacio interno, cerrado en sí mismo, a partir de lo cual lo externo es secundario, dependiente de aquella interioridad. Con ello, bajo el dominio de la consciencia el mundo aparece como posesión de la misma, como contenido que la consciencia toma y apropia. Luego, sosteniendo la dualidad entre sujeto y objeto, se afirma también una distancia entre la consciencia que aparece como propia, de la que puedo dar cuenta, y aquello que ella constituye, que es parte del mundo, de lo exterior. Dicha distancia establece una relación dominada por la interioridad de la consciencia, interioridad que puede aparecer como un ' $\mathrm{Yo}_{\mathrm{o}}$ ', un ego que ante todo da cuenta de sí mismo -como interioridad que aprehende el mundo. Esteban García en Maurice Merleau-Ponty. Filosofía, corporalidad y percepción, afirma que la crítica de Merleau-Ponty al dualismo busca más bien dar cuenta con que hay un estado primario del cuerpo que no radica en la constitución del 'Yo' y lo propio: 
Es en cierto sentido verdadero que mi cuerpo funciona como una máquina ajena a mi conciencia y mi voluntad, como una parte del mundo objetivo, y que yo soy una fuente de pensamientos y decisiones independiente de la máquina. Pero esa no es la única ni la más primaria experiencia que tengo y puedo tener [...] (García 68).

En efecto, puede haber una experiencia del mundo desde un Yo que es causa de toda acción y que se enfrenta a un mundo dado a sí, siendo este Yo el centro al que todo fenómeno se da. Pero este Yo que aparece no reduce el cuerpo a una subjetividad que experimenta para sí, ni el mundo a una objetividad apropiable. Asimismo, en el prólogo de la Fenomenología de la Percepción, Merleau-Ponty nos introduce en esta crítica aludiendo a la idea de un hombre (individuo, 'sujeto') que está en el mundo:

El mundo no es un objeto cuya ley de constitución yo tendría en mi poder; es el medio natural y el campo de todos mis pensamientos y de todas mis percepciones explícitas. La verdad no 'habita' únicamente al 'hombre interior'; mejor aún, no hay hombre interior, el hombre está en el mundo, es en el mundo que se conoce (Merleau-Ponty 1994 10).

Si atendemos a esta descripción del cuerpo, junto con la reflexión de Blanchot vista en el capítulo anterior, podemos establecer que este hombre interior, del cual se habla, no es sino el Yo del día blanchoteano que apropia el mundo. Para sostener la existencia de un hombre interior es necesario abstraer al mismo del mundo en que está inserto y del que se encuentra inmerso: es menester presuponer un aislamiento del Yo frente a un mundo dominable. De la misma manera en que Blanchot caracterizaba la lógica diurna del Yo, en la idea de un 'hombre interior' que constituye el mundo se presenta también aquella con-centración en que el mundo se define, se objetiva y reduce en un eje presupuesto, el del Yo interior.

Luego de plantear el problema del dualismo, Merleau-Ponty comienza paulatinamente a introducir en sus textos su propia visión en torno a la expresividad artística, para finalmente desarrollar una nueva ontología en su escrito inacabado Lo visible y lo invisible. En su ensayo "El entrelazo - El quiasmo", perteneciente a este último escrito, Merleau-Ponty refiere a un fenómeno que radicaliza la idea del cuerpo como unión sujeto-objeto antes presentada en la Fenomenología de la Percepción. Para introducirnos dicho fenómeno, el autor analiza las sensaciones de visión y tacto, con el fin de visibilizar una forma de entender estas experiencias fuera de una dualidad entre vidente y visible (o tocante y tocado ${ }^{4}$ ), anulando con ello la connotación dominante de uno sobre otro: "La

${ }^{4}$ En esta parte del argumento presentado por Merleau-Ponty, se analoga la dinámica de visión y tacto. El autor, de hecho, inicia analizando lo visible, pero luego recurre al fenómeno del tacto para aclarar el fenómeno de la visión. 
mirada, decíamos, envuelve, palpa, abraza las cosas visibles. Como si estuviera con ellas en un acuerdo de armonía preestablecida [...]" (Merleau-Ponty 2010 121). Una mirada que envuelve es una sensación que ya no se concibe frente al mundo. Con esta primera afirmación se hace patente que la inquietud iniciada en la Fenomenología de la Percepción se consuma en este trabajo, a saber, que la unión sujeto-objeto en la experiencia es un hecho, de manera tal que el mundo está envuelto en la mirada como una "armonía preestablecida".

No obstante, Merleau-Ponty agrega un nuevo elemento al fenómeno de esta armonía preestablecida. Considera que si aquello que percibo no se reduce a un mero objeto, este no corresponde a una totalidad cerrada en lo externo y, a su vez, que el cuerpo que percibe tampoco se cierra en un ser-vidente o tocante interno. Con ello, Merleau-Ponty entiende que todo fenómeno debe de antemano estar abierto al mundo sensible: "esto sólo puede suceder si, al mismo tiempo que sentida desde adentro, mi mano es también accesible desde afuera: ella misma tangible, por ejemplo, para mi otra mano; si ella toma lugar entre las cosas que toca" (2010 121-122). En suma, la mano susceptible de ser sintiente y también sentida, puede ser tal en virtud de su existencia en una "Sensibilidad en general" (Id. 128), esta es, la apertura a ser-sensible de todo fenómeno.

Ahora bien, ¿qué implicancia tiene esta Sensibilidad en general para una disolución del Yo, si, de hecho, aún se habla en términos cosas frente a otras (mi mano, tocando la cosa-mano)? Más adelante en este mismo ensayo, Merleau-Ponty hace notar la implicancia de esta Sensibilidad primordial:

Hay visión, tacto, cuando un determinado visible, un determinado tangible se vuelve sobre todo lo visible, todo lo tangible del que forma parte, o cuando súbitamente se encuentra rodeado por él, o cuando, entre él y ellos, y por su intercambio, se forma una Visibilidad, un Tangible en sí que no pertenecen propiamente ni al cuerpo como hecho ni al mundo como hecho (2010 126).

En este fragmento, el autor define la experiencia sensible ya no en virtud de dos elementos opuestos entre sí, sino en virtud de un elemento ontológicamente anterior a todo fenómeno, a saber, una Sensibilidad general, la que replegándose en sí da lugar a una percepción singular; repliegue que es entrelazo, el quiasmo. Dicho quiasmo no es propio ni de una esfera objetual ni de un cuerpo-sujeto constituyente, sino que corresponde más bien a la condición de posibilidad de aquello que reflexivamente se define como cuerpo propio y mundo (sujeto y objeto).

Entonces, cuando el autor da cuenta de que hay algo como mi mirada, y lo visible, está hablando sobre aquellas singularidades que emergen por el repliegue de la sensibilidad. Con ello, al ver desde mi mirada, esta mirada cesa de ser interior y central, y pasa a ser interpelada por una mirada de lo visible. Por ello, más 
adelante Merleau-Ponty afirma que "el vidente, atrapado por lo que ve, no ve más que a sí mismo: hay entonces un narcisismo fundamental de toda visión; $y$, por la misma razón, la visión que él ejerce también la sufre por parte de las cosas" (2010 126). Pero este narcisismo de la visión no significa una amplificación del Yo en lo visto, es decir, no se manifiesta en una apropiación de todo lo visible, sino el movimiento opuesto: la sensibilidad que puede aparecer como propia o interior se ve expropiada desde lo visible, es por esto que el vidente está "atrapado por lo que ve".

Dicho atrape grafica la ausencia de un sujeto: ya no hay dominio de lo visto, ya no hay una libertad de significar lo visto, sino que hay una especie de contramovimiento $^{5}$ desde lo visible hacia la mirada que eventualmente puede reconocerse como mía. En términos blanchoteanos, la percepción ya no está entendida bajo una lógica diurna, en la medida en que ya no sirve al movimiento del Yo, sino que se ha encontrado en el escenario de la percepción un punto de fuga que desestabiliza la centralidad propia del Yo. Por ello, Merleau-Ponty, al ahondar en este contra-movimiento de la sensibilidad, acaba concluyendo una pérdida del quién:

Como lo han expresado muchos pintores, yo me siento mirado por las cosas, mi actividad es idénticamente pasividad -lo cual es el sentido segundo y más profundo del narcisismo: no ver en el afuera como ven los demás, [...], sino sobre todo ser visto a través de él, existir en él, emigrar a él, [...], de manera que vidente y visible se remiten el uno al otro, y ya no se sabe quién ve y quién es visto (2010 126).

La experiencia que Merleau-Ponty testimonia implica más que una relación estrecha con lo pintado, ya que sentirse "mirado por las cosas" designa una actividad que no proviene del cuerpo propio, sino justamente enfrentándose al cuerpo de la mirada. Luego, la mirada del pintor no se concibe meramente inmersa en las cosas, sino afectada por las mismas. Merleau-Ponty introduce esta experiencia para poder pensarla desde la susceptibilidad del cuerpo sensible, ahora abierto a una actividad que interpela.

Por ende, el contra-movimiento de la sensibilidad que se está introduciendo tiene una implicancia directa en cómo se entiende la sensación misma, dado que ya no satisface el definirse como propiedad del cuerpo sensible-como pudo pensarse, por ejemplo, en la ubiestesia o Empfindniss que Husserl introduce en el $\S 36$ de sus

\footnotetext{
${ }^{5}$ Se define como un contra-movimiento en función de una lógica dualista del lenguaje, ya que el 'movimiento' correspondería a aquello que se lleva a cabo desde el sujeto al objeto, ante lo cual el 'contra' movimiento es aquel que se origina en el objeto, las cosas visibles, hacia el sujeto, la mirada. En las notas de trabajo adjuntas en Lo visible y lo invisible - particularmente en una nota fechada en noviembre de 1960-, Merleau-Ponty mismo hace referencia a una 'contra-percepción', como aquello que acompaña a toda percepción (2010 233).
} 
Ideas $I^{6}$. Ahora la sensibilidad introduce una idea diferente del cuerpo, la de uno que no acaba de definirse por su sensibilidad localizada, sino que también por aquellas experiencias que exceden su actividad. Es a propósito de esta consecuencia que Merleau-Ponty menciona la identidad entre la actividad y la pasividad de la mirada, ya que, si hay una 'emigración' a lo visto y se 'existe en' ello, el cuerpo no solo es abandonado en el contra-movimiento de la sensación, es decir, no solo es un cuerpo pasivo que se funde en la sensación que lo interpela, sino que aparece, además, un movimiento que altera esa apertura. En efecto, el que, en principio, haya una mirada que tiende a lo visto, y que lo visto haga "emigrar a él" indica que este cuerpo nunca es pura apertura pasiva a la sensación, ya que siempre hay una tendencia a aquello que interpela: pasivo y activo acaban por anularse entre sí, a partir de un intercambio entre mirada y visible que desdibuja los roles. Aquí, Merleau-Ponty establece una concepción de cuerpo que ya no se define por su rol entre una consciencia abierta al mundo y la objetividad del mundo, sino que en virtud de su relación con aquello que no se hace presente como fenómeno, con aquello que le excede.

Así, no hay principio de acción o reposo en un cuerpo cuya sensibilidad implique el desvanecimiento de la barrera entre la mirada y lo visto. Y sin dicho principio ya no hay centro al cual lo otro se dé: es más, no hay un otro definido al cual otorgar sentido. Luego, el día blanchoteano no tiene cabida en un cuerpo del entrelazo, pues este cuerpo no supone su mismidad, sino que yace abierto a la sensibilidad general que lo envuelve e interpela. Asimismo, si este cuerpo conlleva una simultaneidad paradójica entre pasividad y actividad, y con ello, una suspensión de la subjetividad, es posible identificar en este movimiento las características que Blanchot da para un régimen nocturno, aquel del insomnio, donde consciencia y mundo se infectan ${ }^{7}$ mutuamente en su dispersión indefinida.

Entonces, es posible encontrar en el cuerpo pensado desde el entrelazo o quiasmo aspectos que Blanchot le otorga al sujeto del insomnio, en la medida en que ambos autores captan la presencia de un exceso que implica un conflicto con la subjetividad en el mundo. Y este conflicto tiene que ver con el abandono de la idea de que existe una consciencia yoica en control frente a un mundo dado. En cambio,

\footnotetext{
${ }^{6}$ Fenómeno introducido por Husserl en que da cuenta de la propiedad del cuerpo como portador de sensaciones localizadas (Leib), en función de las sensaciones táctiles de una mano-cosa a otra. En efecto, Husserl lleva a cabo una categorización que distingue dos tipos de cuerpo, uno cósico y otro como carne, a través del ejercicio de dos manos (pertenecientes a un mismo cuerpo) que se tocan entre sí. En dicho ejercicio el autor da cuenta de que una de las manos se siente tocante, ante lo cual aparece como un cuerpo-carne, el que percibe a la otra mano, aquella que, en cambio, aparece como cuerpo-cosa.

7 Noción escogida a propósito de la afirmación de Blanchot en torno al insomnio: de un agotamiento que nos infecta.
} 
tanto el quiasmo como el insomnio presentan a una forma de existencia que desafía la oposición entre actividad y pasividad.

\section{Expresión pictórica y literaria del exceso}

Con Blanchot se puede notar cómo, al problematizar la dualidad activo/pasivo, no solo se presenta una ambigüedad para el sujeto, sino también para la literatura. De un modo similar, Merleau-Ponty da cuenta de que la sensación del mundo en el cuerpo no viene dada por una distancia entre sujeto y objeto, sino justamente borrando dicha oposición, y con ello, problematizando la actividad y pasividad que hay en el cuerpo sensible. Esta observación en Merleau-Ponty se enfatiza con la creación artística, afirmando que ella no se da en virtud de un sujeto yoico, sino por una manera diferente de posicionarse en el mundo. Esto se ilustra con el caso de Matisse: "Aquel mismo pincel que a simple vista saltaba de un acto a otro, se le veía meditar, en un tiempo dilatado y solemne, [...]." y más adelante agrega sobre este meditar que "Matisse se equivocaba si creyó, [...], que había verdaderamente optado, aquel día, entre todos los trazados posibles [...]." (Merleau-Ponty 1964 56). Este fragmento en Signos busca ahondar en cómo se entiende la expresión en el marco de un cuerpo no-yoico, esto es, sin una intencionalidad tematizada de la obra, sino en una relación pre-reflexiva con la creación. Ahora bien, ¿cómo leer la expresión de Matisse desde el insomnio blanchoteano? En la expresión de Matisse puede notarse una determinación que no deja espacio a la duda ni a la elección, determinación que, sin embargo, no es pre-definida a un telos. Es la creación misma la que conduce a Matisse a tales matices y movimientos, sin que haya realmente una voluntad en aquella elección. Así, Tal como el escritor en Blanchot, Matisse se vuelve creador en virtud de su obra, justamente porque ahora la obra es el proceso creativo de un 'autor' que se abandona como tal -motivo por el cual él no opta ni duda. Con ello, si el quiasmo, además de lo que Merleau-Ponty presenta, implica también una ambigüedad entre pasividad y actividad, es menester pensar que la creación artística se lleva a cabo con la participación de un sujeto que está fuera del dominio de lo creado $-\mathrm{O}$, más bien, del creando ${ }^{8}$. Con ello, la expresión artística es el espacio en que el sujeto se suspende en una forma de existencia diferente.

Ahora, ¿cómo se vinculan el insomnio y el sueño en la expresión del pintor y del escritor? Ante todo, es necesario entender la diferencia entre uno y otro. El insomnio en Blanchot sugiere apuntar al aspecto más pasivo de ese estado de consciencia; no obstante una 'pasividad' que requiere de una actividad -en la

\footnotetext{
8 Énfasis debido a que el creando hace referencia al proceso en el cual hay escritura y pintura abierta. En cambio, lo creado puede hacer énfasis en un producto cerrado que es consecuencia de un proceso particular.
} 
medida en que el mundo inmiscuye e interpela a una consciencia (y, quizás, también a un cuerpo) atenta y, a su vez, incapaz. En el insomnio se ilustra una forma de existencia en la cual el mundo ya no se sitúa frente a uno, sino ya calando en la existencia. En cambio, el sueño parece ilustrar una dimensión más activa de esta existencia, en la medida que supone la puesta activa de ella, pero dejando ver una inevitable pasividad, en tanto que no hay una intencionalidad en la expresión que es el sueño.

El sueño es una singularidad en insomnio, un repliegue de lo nocturno, ya que se trata de una expresión con un sujeto ambiguo y descentrado. Dicho sujeto es interpelado por las formas del mundo diurno: se trata de un escritor y un pintor que son interpelados por elementos para-un-sentido (lenguaje y símbolos); no obstante, ellos mismos son tales (escritor y pintor) en la medida en que hay elementos en su escritura y pintura que le impiden confeccionar a su propia voluntad y, más bien, lo sitúan en una producción que subvierte sus voluntades yoicas. Hacia el final de su reflexión sobre el sueño, Blanchot lo caracteriza de la siguiente manera: "El sueño confina con la región donde reina la pura semejanza. [...]. Se busca el modelo original, quisiéramos ser remitidos a un punto de partida, a una revelación inicial, pero no la hay: el sueño es lo semejante que remite eternamente a lo semejante" (2002 238). En este fragmento, Blanchot hace énfasis en que no hay punto de partida, pero partida en tanto que origen fundacional de lo que sucede como sueño. El sueño es una constante remisión entre elementos que Blanchot describe como 'semejantes'; semejantes, pero no idénticos, es decir, el sueño no es mímesis de un origen, ni traducción de algo anterior, sino que consiste en un movimiento propio sin principio ni telos. Entonces, el sueño es la intromisión del estado del insomnio en las formas de la lógica diurna, de manera similar en que, para Merleau-Ponty, la actividad creativa desde el cuerpo-quiasmo supone una re-articulación del mundo en el cual se halla inmerso, sin causa o predeterminación del Yo.

\section{Conclusiones}

De la confrontación entre las anteriores nociones blanchoteanas con el cuerpo en la expresión artística desde Merleau-Ponty, se pueden rescatar ciertas conclusiones. En primer lugar, que el quiasmo que Merleau-Ponty bosqueja en su escrito póstumo puede complementarse con la reflexión que Blanchot realiza acerca del insomnio, en virtud de un elemento común en sus propuestas respectivas, destacado en esta investigación, a saber, que ambas existencias desafían la idea de una subjetividad. Luego, haciendo crítica a esta subjetividad, ambos conducen su planteamiento hacia un cuestionamiento de la dualidad de lo activo y lo pasivo 
como polos absolutos de existencia. En efecto, ambos autores plantean modalidades de existencia que no se definen en virtud de dimensiones sustanciales y determinadas, llámense yo y mundo, sujeto y objeto. Sin embargo, a pesar de no plantear el problema en términos de pasividad y actividad, es posible notar que tanto el insomnio como el quiasmo en Merleau-Ponty suponen una crítica a la dualidad clásica de lo activo-pasivo. Ambos autores plantean una nueva forma de existencia que se halla en una zona gris de aquella dualidad, formulando una experiencia que parece ambigua si se la examina desde lo pasivo-activo.

En segundo lugar, que las similitudes entre ambas posturas en torno al exceso permiten que el gesto blanchoteano de plantear la cuestión de la literatura (cf. Blanchot 2007 271) y la manera en que Merleau-Ponty plantea el ejercicio de la expresión pictórica se amplíen a una cuestión de la expresividad como fenómeno general. Esto es plausible en virtud de que el sujeto ambiguo de la literatura blanchoteana es también un sujeto ambiguo de otras formas de existencia, lo cual se puede afirmar gracias a su propuesta del insomnio y el sueño como momentos de la consciencia, instancias que se presentan de manera tácita en su planteamiento en torno a la escritura. Así, de manera independiente, las propuestas de ambos autores invitan a replantear la experiencia del cuerpo en el ejercicio de la expresión artística, justamente a partir del elemento destacado en esta investigación, a saber, que tanto la consciencia blanchoteana como el cuerpo en Merleau-Ponty suponen una existencia que afirma (siendo activo) a la vez que se halla inmerso en un mundo circundante (siendo pasivo). Así, toda experiencia de ambigüedad, en lo relativo a la relación sujeto-mundo, es susceptible ser analizada a partir de las reflexiones llevadas a cabo por ambos autores, pudiendo encontrar la suspensión del sujeto en instancias diferentes de la escritura y la expresión pictórica.

En suma, es posible dar cuenta de que los movimientos, tanto de MerleauPonty como de Blanchot, se articulan en vías a una suspensión de la figura del sujeto, lo cual llevan a cabo identificando un punto de fuga: el insomnio de la consciencia y el entrelazo del cuerpo, instancias que suspenden la pretendida delimitación de lo subjetivo, abriendo la experiencia a una alteridad, la que frente al sujeto era el mundo, el que no puede simplemente dotársela de sentido. Por ende, el 'sujeto' dual de la expresividad que el pensamiento de Blanchot promueve, a su vez replantear la propuesta que Merleau-Ponty formula en torno al cuerpo sensible: por un lado, presentándonos al cuerpo-quiasmo como un insomnio carnal; $y$, por otro, a una expresividad artística como un sueño encarnado.

Por ende, el punto de fuga es la oportunidad para pensar la expresión artística como un ejercicio constante que nunca se concreta en un objeto, constatando que aquella nunca se cristaliza, sino que siempre está sucediendo. 
Esto, debido a que ya no entendemos este ejercicio como la actividad de un sujeto en vistas a su realización en un objeto, sino que, con el quién definido, sin la división entre cuerpo y mundo, la expresión ocurre en el momento en que estos se tensionan, y ya no por causa de un cuerpo Yo. La expresión, entonces, consiste en la tensión misma, en la experiencia insómnica de dejarse llevar y afirmar en el mundo.

\section{Bibliografía}

Barbaras, Renaud. The Being of the Phenomenon. Merleau.Ponty's Ontology, trads. T. Toadvine, \& L. Lawlor. Bloomington: Indiana University Press, 2004.

Blanchot, Maurice. El espacio literario, trads. V. Palant \& J. Jinkis. Madrid: Nacional, 2002

Blanchot, Maurice. "La literatura y el derecho a la muerte" en La parte del fuego, trad. I. Herrera. Madrid: Arena, 2007. 269-303.

García, Esteban. Maurice Merleau-Ponty. Filosofía, corporalidad y percepción. Editorial Rhesis, 2012.

Merleau-Ponty, Maurice. Fenomenología de la Percepción, trad. J. Cabanes. Barcelona: Ediciones Península, 1994.

Merleau-Ponty, Maurice. Lo visible y lo invisible, trads. E. Consigli, \& B. Capdevielle. Buenos Aires: Nueva Visión, 2010.

Merleau-Ponty, Maurice. 1964. Signos, trads. C. Martínez, \& G. Oliver. Barcelona: Seix Barral, 1964. 